\title{
Simple and accurate one-sided inference from signed roots of likelihood ratios
}

\author{
Thomas J. DICICCIO, Michael A. MARTIN and Steven E. STERN
}

Key words and phrases: Adjusted profile likelihood; ancillary statistic; conditional inference; null hypothesis parametric bootstrap; saddlepoint approximation; similar test.

AMS 1991 subject classifications: Primary 62F05; secondary 62F40.

\begin{abstract}
The authors propose two methods based on the signed root of the likelihood ratio statistic for one-sided testing of a simple null hypothesis about a scalar parameter in the presence of nuisance parameters. Both methods are third-order accurate and utilise simulation to avoid the need for onerous analytical calculations characteristic of competing saddlepoint procedures. Moreover, the new methods do not require specification of ancillary statistics. The methods respect the conditioning associated with similar tests up to an error of third order, and conditioning on ancillary statistics to an error of second order.

RÉSUMÉ

Les auteurs proposent deux méthodes permettant, à partir de la racine signée du rapport des vraisemblances, d'effectuer un test unilatéral d'une hypothèse nulle simple sur un paramètre d'échelle, en présence de paramètres nuisibles. Par le biais de simulations, ces méthodes permettent d'obtenir une précision du troisième ordre tout en évitant les calculs analytiques lourds caractéristiques des méthodes de point de selle concurrentes; elles n'exigent pas non plus la spécification de statistiques libres. Les méthodes proposées respectent, au troisième ordre, le conditionnement associé aux tests similaires, ainsi que celui sur les statistiques libres, mais au deuxième ordre seulement.
\end{abstract}

\section{INTRODUCTION}

Considerable research on improving the accuracy of the standard normal approximation to the distribution of the signed root of the likelihood ratio statistic, $R$, has been conducted. Jensen (1995) provides a detailed review of a wide range of methods, based primarily on saddlepoint approximations, which increase the accuracy in the asymptotic normal approximation to the distribution of $R$ from first to second, or even to third, order. Methods having the latter property typically require specification of a maximal ancillary statistic; see, however, Fraser et al. 
(1999). Such ancillary statistics are readily available in transformation models, and are irrelevant in full-rank exponential families. Outside these two classes of models, ancillary statistics are difficult to specify, and implementation of the most accurate saddlepoint methods can be intractable. These difficulties have prompted the development of methods whose implementations do not depend on ancillaries. Unfortunately, ease of implementation usually has come at the cost of decreased asymptotic accuracy.

The present paper proposes two new methods for inference based on $R$ which are both easily implemented in wide generality, requiring neither the specification of an ancillary nor complex analytical calculations, and are third-order accurate. The first of the new methods (Method 1) employs a standard normal approximation to the distribution of a mean- and variance-corrected version of $R$. DiCiccio \& Stern (1994) provide approximations for the appropriate mean and variance corrections based on asymptotic expansions; however, a straightforward Monte Carlo simulation approach is used here to avoid the need for complex analytical calculations. Location and scale adjustments to $R$ based on asymptotic expansions have been considered by DiCiccio et al. (1990), Efron (1985) and Jensen (1986), among others.

Method 2 is based on tail probabilities of the distribution of $R$ itself, calculated under a choice of parameter value equal to the null hypothesis constrained maximum likelihood estimator. The required tail probability is estimated through Monte Carlo simulation. This new method can be viewed as a null hypothesis parametric bootstrap procedure. The usual parametric bootstrap corresponds to simulation using a parameter value equal to the maximum likelihood estimate. If such an approach is employed, then the resulting inferences are generally only second-order accurate. Thus, the application of Monte Carlo simulation must be employed as in Method 2 in order to achieve third-order accuracy.

Similar methods to those proposed here could be applied in terms of other asymptotically standard normal pivots, such as those based on the Wald or the score statistics. However, two reasons for preferring use of the signed root statistic are asymptotic accuracy and parameterization invariance. When applied with other pivots, Method 1 is accurate only to first order. In contrast, Method 2 offers some advantage over standard methods when used with other pivots, but improves accuracy only to second order. Both Methods 1 and 2, when applied to a parameterization invariant pivot, possess the desirable property of invariance under reparameterizations that preserve the parameter of interest. The signed root statistic is invariant, while the Wald and score statistics generally are not.

Section 2 presents a brief summary of inference procedures based on saddlepoint approximations, while Section 3 introduces the two new methods. Section 4 contains numerical examples that illustrate the accuracy of the new methods. The conditional properties of the proposed methods are discussed in Section 5.

\section{SADDLEPOINT METHODS FOR SIGNED ROOT STATISTICS}

Let $Y=\left(Y_{1}, \ldots, Y_{n}\right)$ be a random vector having a continuous probability distribution that depends on an unknown parameter $\theta=\left(\theta_{1}, \ldots, \theta_{d}\right)$, and denote the $\log$-likelihood function for $\theta$ based on $Y$ by $\ell(\theta)=\ell(\theta ; Y)$. Suppose that $\theta$ is partitioned as $\theta=(\psi, \phi)$, where $\psi=\theta_{1}$ is the scalar parameter of interest and $\phi=\left(\theta_{2}, \ldots, \theta_{d}\right)$ is a $(d-1)$-dimensional nuisance parameter. Let $\hat{\theta}=(\hat{\psi}, \hat{\phi})$ be the global maximum likelihood estimator of $\theta$, and let $\tilde{\theta}_{\psi}=\left(\psi, \tilde{\phi}_{\psi}\right)$ be the constrained 
maximum likelihood estimator of $\theta$ for given value of $\psi$. Standard likelihood inference methods for the parameter $\psi$ handle nuisance parameters by treating the profile likelihood function, $\ell_{p}(\psi)=\ell\left(\tilde{\theta}_{\psi}\right)$, as a genuine log-likelihood. The usual statistic used in testing $\psi=\psi_{0}$ against one-sided alternatives is the signed root of the likelihood ratio statistic,

$$
R\left(\psi_{0}\right)=\operatorname{sgn}\left(\hat{\psi}-\psi_{0}\right)\left\{\ell_{p}(\hat{\psi})-\ell_{p}\left(\psi_{0}\right)\right\}^{1 / 2} .
$$

Under the null hypothesis, $R$ has a standard normal distribution up to an error of $O\left(n^{-1 / 2}\right)$, so that an observed value, $r_{\text {obs }}$, of $R$ has upper one-sided $p$-value $P_{\theta_{0}}\left(R \leq r_{\mathrm{obs}}\right)=\Phi\left(r_{\mathrm{obs}}\right)+O\left(n^{-1 / 2}\right)$, where $\Phi(z)$ denotes the standard normal distribution function.

Using saddlepoint approximations, Barndorff-Nielsen (1986) introduced a quantity $U=U\left(\psi_{0}\right)$ such that $R^{*}=R+R^{-1} \log (U / R)$ has a standard normal distribution up to an error of $O\left(n^{-3 / 2}\right)$. Thus, $p$-values associated with $R^{*}$ are thirdorder accurate. Construction of $U$ requires specification of an ancillary statistic $A$ such that $(\hat{\theta}, A)$ is sufficient. Outside the cases of full-rank exponential families and transformation models, specification of an appropriate ancillary $A$ can be extremely difficult, limiting the usefulness of $R^{*}$. To alleviate the difficulties associated with calculating $U$ for general models, several authors have proposed alternatives quantities $T$, which do not depend on ancillary statistics. Typically, $T=U+O_{p}\left(n^{-1}\right)$, so that the standard normal approximation to the distribution of $R^{\dagger}=R+R^{-1} \log (T / R)$ has an error of $O\left(n^{-1}\right)$, and associated $p$-values are only second-order accurate; see, for example, DiCiccio \& Martin (1993), BarndorffNielsen \& Chamberlin (1994) and Skovgaard (1996).

The profile likelihood function does not possess many of the properties of a genuine log-likelihood. In particular, the bias of the profile score, $E_{\theta}\left\{d \ell_{p}(\psi) / d \psi\right\}$, is $O(1)$. When information about the nuisance parameters is sparse, perhaps because the sample size is small or the number of nuisance parameters is large, using $\ell_{p}(\psi)$ as if it were a true log-likelihood may produce misleading inferences. Consequently, many authors have suggested replacing $\ell_{p}(\psi)$ with an adjusted version, $\bar{\ell}_{p}(\psi)=$ $\ell_{p}(\psi)+b(\psi)$, where $b(\psi)$ is an appropriate adjustment function. Versions of $b(\psi)$ have been developed by Barndorff-Nielsen (1983, 1994), Barndorff-Nielsen \& Cox (1979), Cox \& Reid (1987), DiCiccio et al. (1996), McCullagh \& Tibshirani (1990), and Stern (1997), among others. The proposed adjustment functions $b(\psi)$ typically result in $E_{\theta}\left\{d \bar{\ell}_{p}(\psi) / d \psi\right\}$ being $O\left(n^{-1}\right)$, so that the bias of the adjusted profile score function vanishes asymptotically. Based on an adjusted profile likelihood $\bar{\ell}_{p}(\psi)$, the signed root of the adjusted likelihood ratio statistic for testing the null hypothesis $\psi=\psi_{0}$ against one-sided alternatives is

$$
\bar{R}\left(\psi_{0}\right)=\operatorname{sgn}\left(\bar{\psi}-\psi_{0}\right)\left\{\bar{\ell}_{p}(\bar{\psi})-\bar{\ell}_{p}(\psi)\right\}^{1 / 2},
$$

where $\bar{\psi}$ maximizes $\bar{\ell}_{p}(\psi)$. As for $R$, the distribution of $\bar{R}$ is standard normal up to an error of $O\left(n^{-1 / 2}\right)$, and saddlepoint methods have been used to develop adjusted signed root statistics of the form $\bar{R}^{*}=\bar{R}+\bar{R}^{-1} \log (\bar{U} / \bar{R})$. The distribution of $\bar{R}^{*}$ is standard normal up to an error of $O\left(n^{-3 / 2}\right)$, and its implementation requires specification of an ancillary statistic. Approximations to $\bar{R}^{*}$ of the form $\bar{R}^{\dagger}=$ $\bar{R}+\bar{R}^{-1} \log (\bar{T} / \bar{R})$ have been developed which do not require specification of an ancillary statistic, but the error in the normal approximation to their distribution is $O\left(n^{-1}\right)$. 
For each of the saddlepoint-based methods described in this section, specification of an appropriate ancillary statistic is required in order to achieve third-order accuracy. Alternative procedures developed to avoid this requirement usually only achieve second-order accuracy. Even these procedures require complex analytical calculation, generally involving expectations of higher-order derivatives of the profile likelihood function. In the next section, we discuss in detail two new methods which can achieve third-order accuracy without the need for specification of ancillaries or for complex calculations involving expectations of higher-order derivatives of the profile likelihood.

\section{TWO SIMPLE THIRD-ORDER ACCURATE METHODS}

Lawley (1956) showed that the third- and higher-order cumulants of $R$ are $O\left(n^{-3 / 2}\right)$ or smaller, and that the mean and variance of $R$ can be expanded in the form

$$
E_{\theta}(R)=m(\phi, \phi)+O\left(n^{-3 / 2}\right), \quad \operatorname{var}_{\theta}(R)=1+v(\psi, \phi)+O\left(n^{-3 / 2}\right),
$$

where $m(\psi, \phi)$ is $O\left(n^{-1 / 2}\right)$ and $v(\psi, \phi)$ is $O\left(n^{-1}\right)$. Subject to regularity conditions,

$$
\frac{R-m(\psi, \phi)}{\{1+v(\psi, \phi)\}^{1 / 2}}
$$

has a standard normal distribution up to an error $O\left(n^{-3 / 2}\right)$; see Bickel \& Ghosh (1990).

In most situations, the approximate pivot (1) cannot be used for testing the hypothesis $\psi=\psi_{0}$, since it depends on the value of the nuisance parameter Inference about $\psi$ requires that the nuisance parameter be estimated. Let $\breve{\phi}_{0}$ be an estimator of $\phi$, possibly depending on $\psi_{0}$, such that $\breve{\phi}_{0}-\phi$ is $O_{p}\left(n^{-1 / 2}\right)$ and $E_{\left(\psi_{0}, \phi\right)}\left(\breve{\phi}_{0}\right)=\phi+O\left(n^{-1}\right)$. DiCiccio \& Stern (1994) showed that the third and higher-order cumulants of $R-m\left(\psi_{0}, \breve{\phi}_{0}\right)$ are $O\left(n^{-3 / 2}\right)$ or smaller, while

$$
E_{\left(\psi_{0}, \phi\right)}\left\{R-m\left(\psi_{0}, \breve{\phi}_{0}\right)\right\}=O\left(n^{-3 / 2}\right)
$$

and

$$
\operatorname{var}_{\left(\psi_{0}, \phi\right)}\left\{R-m\left(\psi_{0}, \breve{\phi}_{0}\right)\right\}=1+v_{\breve{\phi}_{0}}\left(\psi_{0}, \phi\right)+O\left(n^{-3 / 2}\right),
$$

where $v_{\breve{\phi}_{0}}\left(\psi_{0}, \phi\right)$ is $O\left(n^{-1}\right)$, its form depending on the choice of estimator, $\breve{\phi}_{0}$. Subject to suitable regularity conditions, the standard normal approximation to the distribution of

$$
\frac{R-m\left(\psi_{0}, \breve{\phi}_{0}\right)}{\left\{1+v_{\breve{\phi}_{0}}\left(\psi_{0}, \breve{\phi}_{0}\right)\right\}^{1 / 2}}
$$

has an error of $O\left(n^{-3 / 2}\right)$. The approximate pivot (2) can be used for inference about $\psi$, and DiCiccio \& Stern (1994) gave general asymptotic formulae for $m\left(\psi_{0}, \phi\right)$ and $v_{\breve{\phi}_{0}}\left(\psi_{0}, \phi\right)$ based on various choices of the estimator $\breve{\phi}_{0}$.

The asymptotic formulae for $m\left(\psi_{0}, \phi\right)$ and $v_{\breve{\phi}_{0}}\left(\psi_{0}, \phi\right)$ are complex expressions involving expectations of high-order derivatives of the log-likelihood. Consequently, it is usually onerous to evaluate them for specific models. A simpler alternative method of calculation is based on Monte Carlo simulation. In general, however, straightforward simulation of $R$ under the choice of model parameter $(\psi, \phi)=$ $\left(\psi_{0}, \breve{\phi}_{0}\right)$ will allow direct estimation of $m\left(\psi_{0}, \breve{\phi}_{0}\right)$ and $v\left(\psi_{0}, \breve{\phi}_{0}\right)$, rather than the 
required $v_{\breve{\phi}_{0}}\left(\psi_{0}, \breve{\phi}_{0}\right)$. The desired quantities could be estimated using an iterative simulation scheme, but such a computationally intensive approach proves unnecessary. For the choice of estimator $\breve{\phi}_{0}=\tilde{\phi}_{0}$, DiCiccio \& Stern (1994) showed that $v_{\tilde{\phi}_{0}}\left(\psi_{0}, \phi\right)=v\left(\psi_{0}, \phi\right)+O\left(n^{-3 / 2}\right)$. Thus,

$$
R_{\mathrm{mv}}=\frac{R-m\left(\psi_{0}, \tilde{\phi}_{0}\right)}{\left\{1+v\left(\psi_{0}, \tilde{\phi}_{0}\right)\right\}^{1 / 2}}
$$

has a standard normal distribution up to an error of $O\left(n^{-3 / 2}\right)$. Hence, $\Phi\left(R_{\mathrm{mv}}\right)$ is an approximate one-sided $p$-value accurate up to an error of $O\left(n^{-3 / 2}\right)$, and the required mean and variance can be estimated through Monte Carlo simulation of $R$ under the choice of model parameter value $(\psi, \phi)=\left(\psi_{0}, \tilde{\phi}_{0}\right)$. This approach we term Method 1.

The results of DiCiccio \& Stern (1994) also can be used to show that, in general,

$$
P_{\left(\psi_{0}, \breve{\phi}_{0}\right)}\left(R \leq r_{\mathrm{obs}}\right)=P_{\left(\psi_{0}, \phi\right)}\left(R \leq r_{\mathrm{obs}}\right)+O\left\{v\left(\psi_{0}, \phi\right)-v_{\breve{\phi}_{0}}\left(\psi_{0}, \phi\right)\right\} .
$$

As a result, tail probabilities of the distribution of the signed root statistic under the choice of model parameter $(\psi, \phi)=\left(\psi_{0}, \breve{\phi}_{0}\right)$ are approximate one-sided $p$-values accurate up to an error of $O\left(n^{-1}\right)$. However, when $\breve{\phi}_{0}=\tilde{\phi}_{0}$,

$$
P_{\tilde{\theta}_{0}}\left(R \leq r_{\mathrm{obs}}\right)=P_{\left(\psi_{0}, \phi\right)}\left(R \leq r_{\mathrm{obs}}\right)+O\left(n^{-3 / 2}\right) .
$$

The tail probability $P_{\tilde{\theta}_{0}}\left(R \leq r_{\text {obs }}\right)$ can be estimated through Monte Carlo simulation. This approach we term Method 2. Methods 1 and 2 coincide up to an error of $O\left(n^{-3 / 2}\right)$. Despite this asymptotic equivalence, the examples of Section 4 suggest that Method 2 often is more accurate than Method 1 in practice.

Analogous results hold for signed roots of adjusted likelihood ratio statistics. For general adjustment functions, DiCiccio \& Stern (1994) showed that the thirdand higher-order cumulants of $\bar{R}$ are $O\left(n^{-3 / 2}\right)$ or smaller, while the mean and variance of $\bar{R}$ satisfy

$$
E_{\left(\psi_{0}, \phi\right)}(\bar{R})=\bar{m}\left(\psi_{0}, \phi\right)+O\left(n^{-3 / 2}\right), \quad \operatorname{var}_{\left(\psi_{0}, \phi\right)}(\bar{R})=1+\bar{v}\left(\psi_{0}, \phi\right)+O\left(n^{-3 / 2}\right),
$$

where $\bar{m}\left(\psi_{0}, \phi\right)$ is $O\left(n^{-1 / 2}\right)$ and $\bar{v}\left(\psi_{0}, \phi\right)$ is $O\left(n^{-1}\right)$. Furthermore, they showed that

$$
\bar{R}_{\mathrm{mv}}=\frac{\bar{R}-\bar{m}\left(\psi_{0}, \tilde{\phi}_{0}\right)}{\left\{1+\bar{v}\left(\psi_{0}, \tilde{\phi}_{0}\right)\right\}^{1 / 2}}
$$

has a standard normal distribution up to an error of $O\left(n^{-3 / 2}\right)$, and they gave asymptotic formulae for $\bar{m}\left(\psi_{0}, \phi\right)$ and $\bar{v}\left(\psi_{0}, \phi\right)$. Therefore, $\Phi\left(\bar{R}_{\mathrm{mv}}\right)$ and $P_{\tilde{\theta}_{0}}(\bar{R} \leq$ $\bar{r}_{\text {obs }}$ ) are both third-order accurate approximate one-sided $p$-values. Both of these quantities can be approximated directly using Monte Carlo simulation of $\bar{R}$ under the choice of parameter $\theta=\tilde{\theta}_{0}$. In other words, Methods 1 and 2 apply to adjusted profile likelihoods as well.

The behaviour of other asymptotically standard normal pivots, such as those based on the Wald or the score statistics, varies from that of $R$ and $\bar{R}$ in that for these pivots, third cumulants are $O\left(n^{-1 / 2}\right)$, fourth cumulants are $O\left(n^{-1}\right)$, and only their fifth and higher-order cumulants are $O\left(n^{-3 / 2}\right)$ or smaller. When applied with such a pivot, Method 1 fails to take skewness properly into account, and an error of $O\left(n^{-1 / 2}\right)$ results. Method 2 is able to account for skewness to some extent, but the resulting error is only $O\left(n^{-1}\right)$. 


\section{EXAMPLES}

Example 1. Normal Regression Model. Suppose $Y_{1}, \ldots, Y_{n}$ follow a normal linear regression model having $q$ regression coefficients and standard deviation $\sigma$, with $\sigma$ the parameter of interest. Cox \& Reid's (1987) adjustment is $b(\psi)=-(1 / 2) q \log F$ in this case and here we employ this function in constructing $\bar{R}$. Following DiCiccio \& Stern (1994),

$$
\begin{array}{ll}
E(R)=-\frac{3 q+2}{(18 n)^{1 / 2}}+O\left(n^{-3 / 2}\right), & \operatorname{var}(R)=1+\frac{3 q+1}{9 n}+O\left(n^{-3 / 2}\right), \\
E(\bar{R})=-\frac{2}{(18 n)^{1 / 2}}+O\left(n^{-3 / 2}\right), & \operatorname{var}(\bar{R})=1+\frac{1}{9 n}+O\left(n^{-3 / 2}\right) .
\end{array}
$$

The quantities $U$ and $\bar{U}$ are available readily in this case.

Table 1: Approximate $p$-values for testing $\sigma=\sigma_{0}$ in normal linear regression; $n=20, q=10$. Table entries are percentages.

\begin{tabular}{ccccccccc}
\hline & \multicolumn{8}{c}{ Exact $p$-value } \\
& $1 \%$ & $2.5 \%$ & $5 \%$ & $10 \%$ & $90 \%$ & $95 \%$ & $97.5 \%$ & $99 \%$ \\
$\Phi(R)$ & $0.0^{4} 57$ & $0.0^{3} 48$ & $0.0^{2} 25$ & 0.014 & 24.740 & 39.142 & 53.021 & 68.530 \\
$\Phi\left(R^{*}\right)$ & 0.418 & 1.194 & 2.655 & 5.938 & 85.650 & 92.435 & 96.044 & 98.335 \\
$\Phi\left(R_{\mathrm{mv},(5)}\right)$ & 1.655 & 0.5670 & 1.441 & 3.672 & 82.308 & 90.374 & 94.821 & 97.744 \\
$\operatorname{Method~1,~} R$ & 0.898 & 2.361 & 4.863 & 9.937 & 89.887 & 94.839 & 97.352 & 98.897 \\
$\Phi(\bar{R})$ & 0.642 & 1.699 & 3.563 & 7.511 & 87.245 & 93.362 & 96.563 & 98.568 \\
$\Phi\left(\bar{R}^{*}\right)$ & 1.000 & 2.499 & 4.997 & 9.993 & 89.987 & 94.992 & 97.495 & 98.998 \\
$\Phi\left(\bar{R}_{\mathrm{mv},(6)}\right)$ & 0.875 & 2.225 & 4.516 & 9.182 & 89.252 & 94.568 & 97.259 & 98.892 \\
$\operatorname{Method~1,R}$ & 1.007 & 2.511 & 5.015 & 10.020 & 90.006 & 95.003 & 97.501 & 99.000 \\
\hline
\end{tabular}

Table 1 exhibits the case $n=20$ and $q=10$; exact one-sided $p$-values are compared with approximate values obtained from $\Phi(R), \Phi\left(R^{*}\right), \Phi(\bar{R})$ and $\Phi\left(\bar{R}^{*}\right)$, as well as with Method 1 applied to both $R$ and $\bar{R}$. The rows in Table 1 labeled $\Phi\left(R_{\mathrm{mv},(6)}\right)$ and $\Phi\left(\bar{R}_{\mathrm{mv},(5)}\right)$ refer to the approximate pivots (3) and (4) applied using the analytical expressions (5) and (6), respectively. Since $R$ and $\bar{R}$ are exactly pivotal in this case, the means and variances of $R$ and $\bar{R}$ do not depend on unknown parameters. As a result, Method 2 yields exact results, up to simulation error. Among the procedures involving $R$, each of $\Phi(R), \Phi\left(R_{\mathrm{mv},(5)}\right)$, and $\Phi\left(R^{*}\right)$ performs poorly, while Method 1 produces acceptable results. The difference between $\Phi\left(R_{\mathrm{mv},(5)}\right)$ and Method 1 is attributable to the poor small-sample performance of the asymptotic formulae $(5)$; these formulae yield $m\left(\psi_{0}, \tilde{\phi}_{0}\right) \approx-1.6865$ and $v\left(\psi_{0}, \tilde{\phi}_{0}\right) \approx 0.1722$, while values obtained from $1,000,000$ Monte Carlo simulations were $m\left(\psi_{0}, \tilde{\phi}_{0}\right) \approx-2.1479$ and $v\left(\psi_{0}, \tilde{\phi}_{0}\right) \approx 0.3203$. Among the methods based on $\bar{R}, \Phi(\bar{R})$ performs poorly, while $\Phi\left(\bar{R}_{\mathrm{mv},(6)}\right)$ performs somewhat better. Both $\Phi\left(\bar{R}^{*}\right)$ and Method 1 applied to $\bar{R}$ provide excellent accuracy. The difference between $\Phi\left(\bar{R}_{\mathrm{mv},(6)}\right)$ and Method 1 applied to $\bar{R}$ arises because of the smallsample inadequacy of the asymptotic formulae (6); the asymptotic formulae give 
$\bar{m}\left(\psi_{0}, \tilde{\phi}_{0}\right) \approx-0.1054$ and $\bar{v}\left(\psi_{0}, \tilde{\phi}_{0}\right) \approx 0.0056$, while the corresponding simulated values were $\bar{m}\left(\psi_{0}, \tilde{\phi}_{0}\right) \approx-0.1511$ and $\bar{v}\left(\psi_{0}, \tilde{\phi}_{0}\right) \approx 0.0114$.

ExAmple 2. Exponential regression. Assume that lifetimes $T_{1}, \ldots, T_{n}$ are independent and exponentially distributed, where $E\left(T_{i}\right)=\exp \left(\alpha+\beta z_{i}\right)$ and $z_{1}, \ldots, z_{n}$ are known covariates. Suppose that interest centers on $\exp \left(\alpha+\beta z_{0}\right)$, the mean lifetime for covariate value $z_{0}$ and let $\psi=\alpha+\beta z_{0}$. The log-likelihood function for $\psi$ and $\beta$ is $\ell(\psi, \beta)=-n \psi-n \beta \bar{c}-e^{-\psi} \sum T_{i} \exp \left(-\beta c_{i}\right)$, where $c_{i}=z_{i}-z_{0}$ $(i=1, \ldots, n)$ and $\bar{c}=n^{-1} \sum c_{i}$. The maximum likelihood estimators, $\hat{\psi}$ and $\hat{\beta}$, satisfy the equations

$$
\hat{\psi}=\log \left\{n^{-1} \sum T_{i} \exp \left(-\hat{\beta} c_{i}\right)\right\}, \quad \sum T_{i}\left(c_{i}-\bar{c}\right) \exp \left(-\hat{\beta} c_{i}\right)=0,
$$

and the constrained maximum likelihood estimator, $\tilde{\beta}_{0}$, is the solution to the equation $\sum T_{i} c_{i} \exp \left(-\tilde{\beta}_{0} c_{i}\right)=n \bar{c} e^{\psi_{0}}$. The signed root of the likelihood ratio statistic is

$$
R=\operatorname{sgn}\left(\hat{\psi}-\psi_{0}\right)\left[2 n\left\{\left(\psi_{0}-\hat{\psi}\right)+\left(\tilde{\beta}_{0}-\hat{\beta}\right) \bar{c}+n^{-1} e^{-\psi_{0}} \sum T_{i} \exp \left(-\tilde{\beta}_{0} c_{i}\right)-1\right\}\right]^{1 / 2} .
$$

Again, the quantity $U$ is available readily in this case.

Table 2: Simulated lower tail probabilities of approximate one-sided $p$-values for $\psi=\alpha+\beta \log _{10}(50000) ; n=5$. Table entries are percentages.

\begin{tabular}{ccccccccc}
\hline & & \multicolumn{7}{c}{ Nominal Size } \\
& & & & & & \\
& $1 \%$ & $2.5 \%$ & $5 \%$ & $10 \%$ & $90 \%$ & $95 \%$ & $97.5 \%$ & $99 \%$ \\
$\Phi(R)$ & 0.50 & 1.34 & 2.91 & 6.32 & 85.60 & 92.35 & 95.97 & 98.29 \\
$\Phi\left(R^{*}\right)$ & 0.98 & 2.49 & 5.01 & 9.99 & 90.04 & 94.97 & 97.49 & 99.00 \\
Method 1, R & 1.03 & 2.56 & 5.10 & 10.10 & 90.07 & 95.04 & 97.51 & 99.00 \\
Method 2, $R$ & 0.98 & 2.48 & 5.02 & 9.99 & 90.06 & 94.97 & 97.48 & 98.96 \\
& & & & & & & & \\
\hline
\end{tabular}

Lawless (1982, example 6.3.2) used an exponential regression model to analyze Feigl \& Zelen's (1965) leukemia data concerning the survival time in weeks of 17 patients. For these data, the covariate $z$ is the base- 10 logarithm of initial white blood cell count. Table 2 concerns the subset of size 5 from these data consisting of lifetimes 156, 108, 143, 1, 65 and corresponding covariate values 2.88, 4.02, 3.85, 5,5 . Suppose that the mean lifetime for $z_{0}=\log _{10}(50,000)$ is of interest. Table 4 shows the lower tail probabilities of the null distribution for approximate $p$-values for one-sided tests for $\psi$, obtained from $\Phi(R), \Phi\left(R^{*}\right)$, and Methods 1 and 2, based on 5 observations from an exponential regression model having these covariate values. The tail probabilities were estimated based on 250,000 random datasets. For Method 1, 500 Monte Carlo simulations were used to estimate the mean and variance corrections, while for Method 2, 2000 Monte Carlo simulations were used to estimate the required tail probability. Results obtained from $\Phi(R)$ are poor, while Methods 1 and 2 and $\Phi\left(R^{*}\right)$ each performs well.

For the preceding examples, implementation of the most accurate saddlepoint methods has been straightforward and their accuracy is excellent. In these situations, Methods 1 and 2 have little added benefit. Nevertheless, their implementation is simple and the fact that their performance is competitive with that 
of saddlepoint methods is encouraging for cases where saddlepoint methods are difficult or impossible to implement.

EXAmple 3. Variance component model. Skovgaard (1996) considered the oneway model $Y_{i j}=\psi+\alpha_{i}+e_{i j}\left(i=1, \ldots, m ; j=1, \ldots, n_{i}\right)$, where the $\alpha_{i}$ 's and the $e_{i j}$ 's are all independent normal variables having mean 0 and variances $\sigma_{\alpha}^{2}$ and $\sigma_{e}^{2}$, respectively. When the group sizes $n_{1}, \ldots, n_{m}$ are not all equal, the maximum likelihood estimators do not have closed form expressions, and the likelihood equations must be solved iteratively.

Table 3: Simulated coverage levels of approximate symmetric two-sided confidence intervals for $\psi ; n=15$. Table entries are percentages.

\begin{tabular}{ccccc}
\hline & \multicolumn{4}{c}{ Nominal coverage probability } \\
& $80 \%$ & $90 \%$ & $98 \%$ & $99.8 \%$ \\
$\Phi(R)$ & 72.05 & 83.55 & 95.10 & 99.15 \\
$\Phi(\tilde{R})$ & 79.77 & 89.87 & 97.90 & 99.78 \\
Satterthwaite & 80.05 & 90.13 & 98.10 & 99.82 \\
Method 1, $R$ & 79.77 & 89.91 & 98.04 & 99.82 \\
Method 2, $R$ & 79.86 & 89.93 & 97.97 & 99.77 \\
\hline
\end{tabular}

For this model, ancillary statistics are not available to determine $U$. Versions of $T$ also prove difficult to obtain. In contrast, Methods 1 and 2 are applied easily. Skovgaard performed a simulation study demonstrating the accuracy of $\tilde{R}$, his large-deviation version of $R^{\dagger}$, when $m=5, n_{i}=i, \sigma_{e}=0.04$, and $\sigma_{\alpha}=1$, and he compared his method with the Satterthwaite approximation. Since this problem is symmetric about $\psi=0$, Skovgaard gave estimated coverages of two-sided confidence intervals. His results are reproduced in Table 3, along with corresponding two-sided results for Methods 1 and 2. All of the coverages reported in Table 3 are based on 100,000 random datasets. In applying each of Methods 1 and 2, 2000 Monte Carlo simulations were used. Skovgaard noted that both Satterthwaite's approximation and $\Phi(\tilde{R})$ produced accurate approximations. Table 3 shows that the proposed methods also produce comparable approximations.

\section{CONDITIONAL INFERENCE}

When $\theta$ is the canonical parameter of an exponential family, a similar test of the null hypothesis $\psi=\psi_{0}$ is generated through conditioning on $\tilde{\phi}_{0}$. In this context, Jensen (1986) showed that $R_{\mathrm{mv}}$ defined by (3) has a standard normal distribution up to an error of $O\left(n^{-3 / 2}\right)$ conditionally as well as unconditionally. Thus, the approximate one-sided $p$-values produced by Methods 1 and 2 for $R$ are conditionally third-order accurate. Furthermore, Skovgaard (1987) showed that $R^{*}$ has a standard normal distribution up to an error of $O\left(n^{-3 / 2}\right)$ conditionally as well as unconditionally. From the perspective of similar tests, the conditional properties of Methods 1 and 2 when applied to $R$ are asymptotically equivalent to those of $\Phi\left(R^{*}\right)$. The same properties hold for $\Phi\left(\bar{R}^{*}\right)$ and for Methods 1 and 2 applied to $\bar{R}$; see Fraser et al. (1991) and DiCiccio \& Martin (1991).

Ancillary statistics do not arise in the context of full-rank exponential families; however, they are a key component of inference for models outside this class. 
Approximate procedures should take reasonable ancillary statistics into account. Barndorff-Nielsen (1986) showed that the conditional distribution of $R^{*}$ given an ancillary $A$ is standard normal up to an error of $O\left(n^{-3 / 2}\right)$. In contrast, the calculations of McCullagh (1987) and DiCiccio \& Martin (1993) indicate that the conditional distribution of $R_{\mathrm{mv}}$ given $A$ is standard normal up to an error of $O\left(n^{-1}\right)$. The same order of conditional accuracy holds for Methods 1 and 2 when applied to $R$. In those cases where $R^{*}$ is available, results from $\Phi\left(R^{*}\right)$ should have better conditional properties than the results from Methods 1 or 2 , although the unconditional performances of $\Phi\left(R^{*}\right)$ and Methods 1 and 2 should be similar.

Example 3 (Continued). Exponential regression. For the exponential regression model, conditional inference given ancillary statistics $\log T_{i}-\hat{\psi}-\hat{\beta} c_{i}(i=1, \ldots, n)$ is described by Lawless (1982, section 6.3.2). Based on the 5 observations considered earlier, Table 4 allows comparison between exact conditional significance levels with approximate levels obtained from $\Phi(R), \Phi\left(R^{*}\right)$, and Methods 1 and 2, for a variety of values of $\psi_{0}$. Monte Carlo simulation sizes of 50,000 and 200,000 were used for Methods 1 and 2, respectively. All three higher-order procedures offer substantial improvement over $\Phi(R)$. However, $\Phi\left(R^{*}\right)$ performs slightly better than either of the new methods, indicating a slight advantage in conditional accuracy despite the results of Table 2 where no significant difference between the unconditional accuracy of these methods was found.

Table 4: Exact conditional and approximate $p$-values for testing $\psi_{0}=\hat{\psi}-\delta ; n=5$. Table entries are percentages.

\begin{tabular}{cccccc}
\hline$\delta$ & Conditional level & $\Phi(R)$ & $\Phi\left(R^{*}\right)$ & Method 1 & Method 2 \\
-2.05 & 0.600 & 0.339 & 0.589 & 0.639 & 0.612 \\
-1.85 & 1.066 & 0.621 & 1.047 & 1.129 & 1.076 \\
-1.50 & 2.789 & 1.723 & 2.747 & 2.927 & 2.860 \\
-1.30 & 4.691 & 3.004 & 4.627 & 4.895 & 4.851 \\
-0.95 & 10.916 & 7.479 & 10.798 & 11.277 & 11.170 \\
0.55 & 90.468 & 85.843 & 90.410 & 90.743 & 90.694 \\
0.70 & 94.996 & 92.035 & 94.964 & 95.177 & 95.142 \\
0.85 & 97.728 & 96.099 & 97.714 & 97.833 & 97.744 \\
1.00 & 99.132 & 98.381 & 99.126 & 99.183 & 99.132 \\
1.10 & 99.591 & 99.191 & 99.588 & 99.619 & 99.560 \\
& & & & & \\
\hline
\end{tabular}

This advantage in conditional asymptotic accuracy adds credence to the suggestion that when $R^{*}$ is readily available, it is preferable to Methods 1 and 2. However, in other situations, the simplicity of their implementation and their third-order asymptotic accuracy make Methods 1 and 2 appealing. Moreover, the various versions of $R^{\dagger}$ and $\bar{R}^{\dagger}$, which do not depend on ancillary statistics, typically have a standard normal distribution up to an error of $O\left(n^{-1}\right)$ both conditionally and unconditionally. Thus, the conditional properties of Methods 1 and 2 are asymptotically the same as those of $\Phi\left(R^{\dagger}\right)$ and $\Phi\left(\bar{R}^{\dagger}\right)$. In a compelling location-scale example, McCullagh (1992) showed that conditional $p$-values based on distinct ancillary statistics differ by $O\left(n^{-1}\right)$. His results suggest insisting that inference procedures be conditionally third-order accurate is unwarranted. 


\section{CONCLUDING REMARKS}

The accuracy of the new methods is clearly dependent on the number of Monte Carlo simulations used. The simulation sizes used in the examples here were chosen based on experience and are designed to ensure that the simulation error is a negligible portion of the overall coverage errors. In general, for both Methods 1 and 2 a simulation size of $B$ leads to simulation errors which are $O\left(B^{-1}\right)$. Choice of a suitable simulation size can be based on this fact. Of course, it is commonly accepted that means and variances require fewer simulations for precise estimation than do tail probabilities. In this sense, Method 1 can be seen to be less computationally intensive than Method 2.

Finally, the asymptotic orders of the error terms asserted here pertain to moderate-deviation regions. There has recently been considerable interest in the largedeviation properties of higher-order approximations. In exponential family problems, the standard normal approximation to the distribution of $R^{*}$ has a relative error of $O\left(n^{-1}\right)$ in the large-deviation region; see, for example, Jensen (1992) and Skovgaard (1996). The standard normal approximation to the distribution of Skovgaard's (1996) version of $R^{\dagger}$ has a relative error of $O\left(n^{-1 / 2}\right)$ in the large-deviation region, where $R$ itself is $O\left(n^{1 / 2}\right)$. In contrast, other versions of $R^{\dagger}$ fail to have this desirable large-deviation property. The standard normal approximation for $R_{\mathrm{mv}}$ cannot be expected to have relative error converging to 0 in the large-deviation region. As a result, it is unlikely that Method 1 has this desirable property. Of course, it is possible that Method 2 behaves more appropriately in the large-deviation region. Thus, it might be possible to distinguish between Methods 1 and 2 based on their large-deviation behaviour, and this topic deserves further attention.

\section{ACKNOWLEDGEMENTS}

We are grateful to participants at workshops held at Brixen, Italy and Banff, Canada for their helpful comments, especially Anthony Davison, Bradley Efron, Jens Jensen, Peter McCullagh, Don Pierce, Judith Rousseau, Tom Severini, and Ib Skovgaard. The computing assistance of Anna Clara Monti and Russell Zaretzki is also gratefully acknowledged.

\section{REFERENCES}

O. E. Barndorff-Nielsen (1983). On a formula for the distribution of the maximum likelihood estimator. Biometrika, 70, 343-365.

O. E. Barndorff-Nielsen (1986). Inference on full or partial parameters based on the standardized signed log likelihood ratio. Biometrika, 73, 307-322.

O. E. Barndorff-Nielsen (1994). Adjusted versions of profile likelihood and directed likelihood, and extended likelihood. Journal of the Royal Statistical Society Series $B, 56,125-140$.

O. E. Barndorff-Nielsen \& S. R. Chamberlin (1994). Stable and invariant adjusted directed likelihoods. Biometrika, 81, 485-500.

O. E. Barndorff-Nielsen \& D. R. Cox (1979). Edgeworth and saddlepoint approximations with statistical applications (with discussion), Journal of the Royal Statistical Society Series B, 41, 279-312.

P. J. Bickel \& J. K. Ghosh (1990). A decomposition for the likelihood ratio statistic and the Bartlett correction - a Bayesian argument. Annals of Statistics, 18, 1070-1090. 
D. R. Cox \& N. Reid (1987). Parameter orthogonality and approximate conditional inference (with discussion). Journal of the Royal Statistical Society Series B, 49, $1-39$.

T. J. DiCiccio, C. A. Field \& D. A. S. Fraser (1990). Approximations of marginal tail probabilities and inference for scalar parameters. Biometrika, 77, 77-95.

T. J. DiCiccio \& M. A. Martin (1991). Approximations of marginal tail probabilities for a class of smooth functions with applications to Bayesian and conditional inference. Biometrika, 78, 891-902.

T. J. DiCiccio \& M. A. Martin (1993). Simple modifications for signed roots of likelihood ratio statistics. Journal of the Royal Statistical Society Series B, 55, 305-316.

T. J. DiCiccio, M. A. Martin, S. E. Stern \& G. A. Young (1996). Information bias and adjusted profile likelihoods. Journal of the Royal Statistical Society Series B, 58, 189-203.

T. J. DiCiccio \& S. E. Stern (1994). Constructing approximately standard Normal pivots from signed roots of adjusted likelihood ratio statistics. Scandinavian Journal of Statitics, 21, 447-460.

B. Efron (1985). Bootstrap confidence intervals for a class of parametric problems. Biometrika, 86, 258-265.

P. Feigl \& M. Zelen (1965). Estimation of exponential survival probabilities with concomitant information. Biometrics, 21, 826-838.

D. A. S. Fraser, N. Reid \& A. Wong (1991). Exponential linear models: a two-pass procedure for saddlepoint approximation. Journal of the Royal Statistical Society Series B, 53, 483-492.

D. A. S. Fraser, N. Reid \& J. Wu (1999). A simple general formula for tail probabilities for frequentist and Bayesian inference. Biometrika, 86, 249-264.

J. L. Jensen (1986). Similar tests and the standardized log likelihood ratio statistic. Biometrika, 73, 567-572.

J. L. Jensen (1992). The modified signed likelihood statistic and saddlepoint approximations. Biometrika, 79, 693-703.

J. L. Jensen (1995). Saddlepoint Approximations. Oxford University Press, Oxford, United Kingdom.

J. F. Lawless (1982). Statistical Models and Methods for Lifetime Data. Wiley, New York.

D. N. Lawley (1956). A general method for approximating to the distribution of the likelihood ratio criteria. Biometrika, 43, 295-303.

P. McCullagh (1987). Tensor Methods in Statistics. Chapman and Hall, London.

P. McCullagh (1992). Conditional inference and Cauchy models. Biometrika, 79, 247260.

P. McCullagh \& R. J. Tibshirani (1990). A simple method for the adjustment of profile likelihoods. Journal of the Royal Statistical Society Series B, 52, 325-44.

I. M. Skovgaard (1987). Saddlepoint expansions for conditional distributions. Journal of Applied Probability, 24, 875-887.

I. M. Skovgaard (1996). An explicit large-deviation approximation to one-parameter tests. Bernoulli, 2, 145-165.

S. E. Stern (1997). A second-order adjustment to the profile likelihood in the case of a multidimensional parameter of interest. Journal of the Royal Statistical Society Series B, 59, 653-665. 
Received 5 May 1999

Accepted 14 March 2000
Thomas J. DiCICCIO: tjd9@cornell .edu Department of Social Statistics

Cornell University Ithaca, NY 14853

USA

Michael A. MARTIN: Michael.Martin@anu.edu.au Department of Statistics and Econometrics The Australian National University Canberra, ACT 0200 Australia

Steven E. STERN: Steven.Stern@anu.edu.au Department of Statistics and Econometrics The Australian National University Canberra, ACT 0200 Australia 